\title{
Rock walls: small-scale diversity hotspots in the subtidal Gulf of Maine
}

\author{
Robert J. Miller ${ }^{1,2, *}$, Ron J. Etter ${ }^{1}$ \\ ${ }^{1}$ Department of Biology, University of Massachusetts Boston, Boston, Massachusetts 02125 USA \\ ${ }^{2}$ Present address: Marine Science Institute, University of California, Santa Barbara, California 93106 USA
}

\begin{abstract}
The physical orientation of rocky substrate profoundly affects subtidal marine communities of sessile organisms. Anecdotal descriptions of dramatic differences between communities on rock walls and adjacent horizontal rocky bottoms abound in the literature and are common knowledge among scuba divers, yet these differences have rarely been quantified by ecologists. We sampled rock walls and adjacent horizontal rock bottoms at 8 subtidal sites across the Gulf of Maine. Species richness and abundance, in percent cover, of sessile invertebrates on vertical walls averaged 4 times higher than that on horizontal platforms, whereas abundance of macroalgae on horizontal rock was about 3 times that on vertical walls. Both macroalgae and sessile invertebrates were less abundant, particularly on horizontal surfaces, at sites with high sea urchin densities. The consistency of sessile invertebrate domination of vertical walls versus macroalgal domination of horizontal rock, combined with previous experimental results, suggests that competition for space between autotrophic and heterotrophic organisms drives this pattern. The partitioning of autotrophs (macroalgae) and heterotrophs (sessile invertebrates) between horizontal and vertical surfaces respectively implies that topographic heterogeneity plays an important role in the structure, composition and function of rocky subtidal ecosystems.
\end{abstract}

KEY WORDS: Rock walls · Sessile invertebrates $\cdot$ Gulf of Maine · Diversity $\cdot$ Grazing

\section{INTRODUCTION}

Topography is a common source of spatial heterogeneity that may profoundly influence species distributions and is often associated with ecological zonation patterns. In terrestrial systems, elevation, slope, and aspect shape plant communities at the landscape scale (e.g. Whittaker 1960, Clark et al. 1995, Ohmann \& Spies 1998), and influence the distribution and abundance of animals, particularly small ectotherms (Weiss et al. 1988, Porter et al. 2002, Beck \& Jennings 2003). Slope of the shore shapes intertidal community zonation by altering the area of tidal influence, thermal stress, and wave shock (Ricketts et al. 1985, Denny 1988, Little \& Kitching 1996, Bertness 1999). In marine rocky subtidal communities, substrate slope may be a basic determinant of community structure: horizontal rock or gentle slopes are often dominated by macroalgae, while vertical rock walls are cov- ered by epifaunal suspension-feeding invertebrates (reviewed in Witman \& Dayton 2001, Miller \& Etter 2008). This pattern has been observed on rocky subtidal bottoms all over the world (e.g. Eastern Pacific: Pequegnat 1964, Vance 1988, Baynes 1999; Northwest Atlantic, including the Gulf of Maine: Noble et al. 1976, Witman \& Cooper 1983, Sebens 1986a,b, Mathieson et al. 1991; Northeast Atlantic: Lundalv 1971; Caribbean: Bruno \& Witman 1996; Mediterranean: Weinberg 1978, Uriz et al. 1998; New Zealand: Witman \& Grange 1998; Australia: Irving \& Connell 2002, Knott et al. 2004; Galapagos, Seychelles, South Africa, Palau, Antarctica and Chile: Witman et al. 2004). The pattern has rarely, however, been quantified (but see Knott et al. 2004). Quantifying community patterns is necessary to focus attention on, and generate viable hypotheses about the processes that influence patterns of species distribution and abundance (Underwood et al. 2000). 
Despite general observations of dramatic differences in adjacent subtidal communities on vertical and horizontal substrates, the extent of these patterns and the processes that produce them are poorly understood. The processes responsible may include: (1) shading by vertical walls, which negatively affects algal growth and potentially attracts photonegative invertebrate larvae (e.g. Sebens 1986a, Gotelli 1987, Gili \& Coma 1998, Miller \& Etter 2008); (2) lack of physical and biological disturbance on walls (Witman \& Cooper 1983, Sebens 1986a, Vance 1988); (3) lack of sedimentation on walls, thus freeing suspension feeders from clogged filtration and respiratory systems (Weinberg 1978, Witman \& Sebens 1990, Irving \& Connell 2002); and (4) relatively higher food and larval flux to walls due to increased water flow (Leichter \& Witman 1997, Gili \& Coma 1998, Genovese \& Witman 1999). Patches of high invertebrate abundance on walls, once established, may be maintained by enhanced local recruitment of short-lived larvae (Graham \& Sebens 1996, Smith \& Witman 1999). Experiments implicate shading as an important factor shaping the pattern, with competition with algae being the most likely mechanism inhibiting invertebrates on horizontal surfaces (Irving \& Connell 2002, Miller \& Etter 2008); although physical disturbance (Witman \& Cooper 1983, Miller \& Etter 2008), and urchin grazing, where urchins are abundant (Sebens 1986a), might also exert strong influences. To establish the generality of this substrateangle pattern, particularly with respect to species richness (but see Knott et al. 2004), and better identify the forces that might shape them, we need to systematically quantify differences in the structure and composition of epifaunal communities on vertical and horizontal surfaces.

Gradients in the processes shaping the substrate-angle pattern might also generate larger-scale patterns. An example lies in a classic subtidal zonation scheme: the infralittoral zone extends from the low tide mark to the critical depth for photosynthesis, and is dominated by macroalgae, whereas the deeper circalittoral zone is dominated by sessile and mobile invertebrates (Golikov \& Scarlato 1968, Dayton 1975). This 'depth emergence' of invertebrates is generally explained as an effect of decreasing light with depth, and a concomitant decrease in macroalgal competitors, combined with decreasing physical disturbance from surface waves with depth (reviewed in Witman \& Dayton 2001). Study of the sharp transitions in processes that occur with changes in substrate angle may lead to a greater understanding of these larger-scale patterns.

To test whether substrate angle affects epifaunal community structure we compared diversity and coverage of subtidal sessile invertebrates on vertical versus horizontal rock surfaces at exposed sites 10 to $12 \mathrm{~m}$ deep in the Gulf of Maine (GOM). Based on previous research (cited above), we predicted that diversity and coverage of invertebrates would be greater on vertical surfaces. To test the validity of various processes suggested as possible mechanisms mediating differences between vertical and horizontal surfaces, we compared the relative abundances of sessile invertebrates, macroalgae and grazers. The specific secondary predictions we considered are as follows. (1) Diversity and abundance of sessile invertebrates on both vertical and horizontal surfaces, but particularly the latter, will be lower at sites with higher densities of omnivorous sea urchins (Strongylocentrotus droebachiensis Müller). (2) Invertebrate diversity on horizontal substrates will be positively related to that on nearby vertical walls, due to restricted dispersal of many sessile invertebrate larvae. We also describe patterns of abundance of other grazers (limpets, chitons) and predators (asteroids) vis-à-vis substrate angle.

\section{MATERIALS AND METHODS}

Study sites and sampling. We sampled 8 sites in the GOM (Fig. 1), all on exposed rocky coasts with abun-

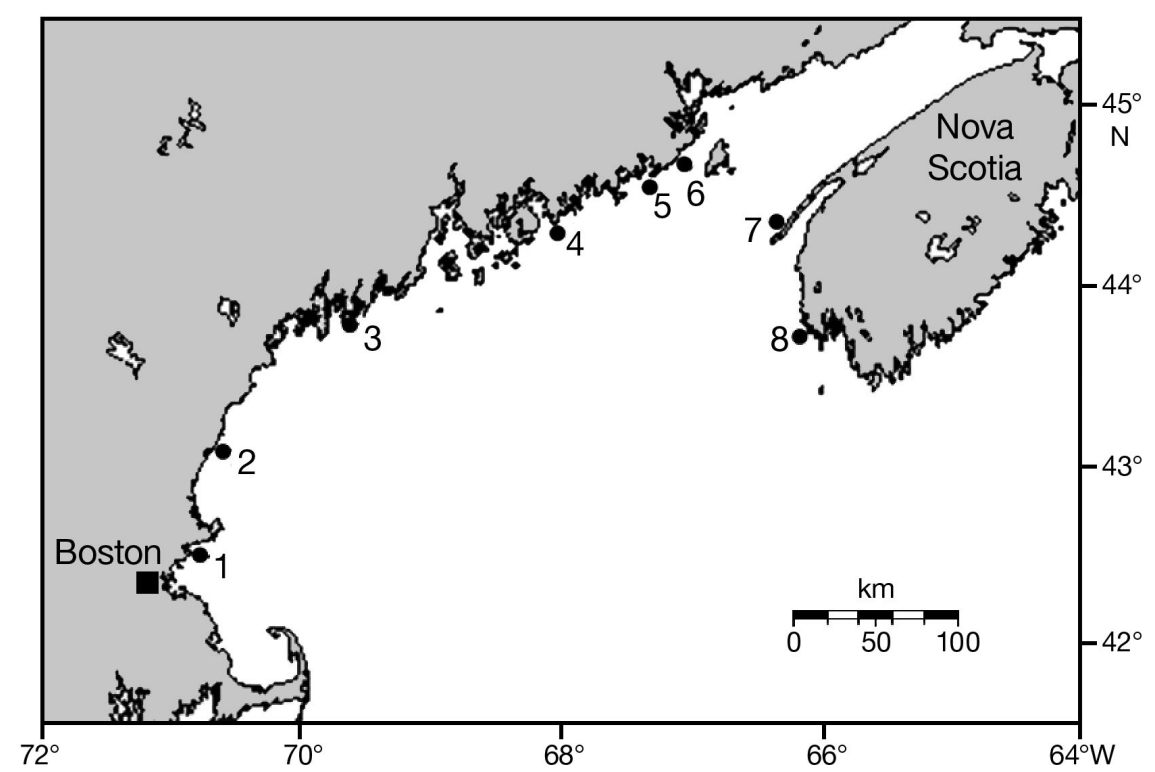

Fig. 1. Map of the Gulf of Maine showing location of survey sites. 1: Shag Rocks (SR), 2: Wood Island (WI), 3: Thrumcap (TC), 4: Bunker Point (BP), 5: Moose Cove (MC), 6: Cutler (CR), 7: Whale Cove (WC), 8: Pubnico (PU) 
dant vertical and horizontal granitic rock substrate, during August and September 2003. Sampling was conducted at 10 to $12 \mathrm{~m}$ depth below mean low tide. Community structure was quantified from photoquadrats $\left(0.25 \mathrm{~m}^{2}\right)$ positioned at random points marked on a $50 \mathrm{~m}$ transect tape that was laid out along the first vertical wall encountered. If the wall ended before enough photos were taken, we swam to the next nearest wall and continued. All photos were taken along the vertical midsection of walls. Wall height was not measured, although most were $\sim 1.5$ to $3 \mathrm{~m}$ high. Horizontal substrate was photographed in the same way with a $50 \mathrm{~m}$ tape stretched out in a haphazard direction. 18 to 36 photos per orientation were taken with a Nikonos camera (15 mm lens, Fuji Velvia 50 ASA slide film) mounted on a quadropod (Witman 1987, Coyer et al. 1999). Large algae (e.g. kelp blades) were trimmed with shears before photographing if necessary to clear the view of the substrate. Specimens of invertebrates were preserved and identified to confirm photo identifications when necessary. To evaluate whether the presence of low-profile algae was significantly affecting percent cover and diversity estimates of invertebrates on horizontal substrates, we removed all algae by hand from 10 quadrats at the Shag Rocks site and rephotogaphed them for comparison. Diversity and percent cover for these slides was measured as described below.

Because estimates of species richness are dependent on sample size, we analyzed photoquadrats until we reached the asymptote of the species accumulation curve. At the asymptote, comparisons of species richness should be independent of sampling effort. To approximate the asymptote, 18 slides per orientation (vertical and horizontal) were generally sufficient. Photoquadrats were examined under a dissecting microscope and projected on a screen to visually quantify the presence or absence of sessile invertebrates. Species were identified as finely as possible with the aid of voucher specimens and additional close-up photos. When the distinction between 2 possibly separate species was in doubt, they were lumped together to avoid overestimation of richness.

Percent cover was estimated by projecting each slide onto a screen with 100 random dots and assigning what fell under each dot to one of the following categories: crustose coralline algae, Peysonnelia sp. (soft red crustose algae), macroalgae, algal turf, tube complex (consolidated mats of tubes built by amphipods and polychaetes, as described in Sebens 1986a), sessile invertebrates, the mussels Mytilus sp. and Modiolus modiolus Linnaeus, sediment, and bare rock. For analysis, macroalgae and algal turf, which was often intermixed and difficult to separate, was grouped under macroalgae. Mussels were analyzed separately from other sessile invertebrates, due to their typical distribution on horizontal substrates (Witman 1987). Grazers and predators were counted, including the urchin Strongylocentrotus droebachiensis, the limpet Tectura (=Acmaea) testudinalis Müller, the chitons Tonicella spp. and Stenosemus (=Ischnochiton) albus Linnaeus, and the asteroids Asterias forbesi Desor, A. vulgaris Verrill and Henricia sanguinolenta Müller.

Statistical analysis. Species accumulation curves were generated using EstimateS v. 6.0b1 software (Colwell 1997). Accumulation curves were plotted as both the number of species observed $\left(S_{\text {obs }}\right)$ and Chao2, which better estimates true richness for small samples, taking rare species into account, as follows: Chao $2=S_{\text {obs }}+$ $\left(Q_{1}\right)^{2} / 2\left(Q_{2}\right)$, where $S_{\text {obs }}$ is the number of species observed in the pooled samples, $Q_{1}$ is the number of species occurring in only one sample (singletons), and $Q_{2}$ is the number occurring in 2 samples (doubletons) (Colwell \& Coddington 1994). Richness ( $S_{\text {obs }}$ and Chao2) was compared on horizontal versus vertical surfaces using 1-way ANOVA with orientation (horizontal vs. vertical) as a fixed factor. Mantel tests (R v. 2.91.1, www.r-project.org/) were used to test whether the number of species shared between sites was related to the geographic distance separating samples. Beta diversity between sites was compared using 3 metrics: $S_{\text {obs }}$ shared, the Bray-Curtis index (Magurran 2004), and Chao2 shared estimated (estimated number of species shared by first and second samples, Chen et al. 1995). Distances (km) between all site pairs were calculated using the geodistances program in the R software package (Casgrain \& Legendre 2001).

We explored differences in community structure between sites and orientations using non-metric multidimensional scaling (NMDS) based on the Bray-Curtis distance metric, and with canonical analysis of principal coordinates (CAP), using a permutation test of significance of differences between groups, and the 'leave-one-out' approach to estimate goodness of fit of the groups. Correlation coefficients of the canonical axis with the original taxonomic categories were used to identify the species most influencing the ordination pattern (Anderson \& Robinson 2003, Anderson \& Willis 2003). To compare the multivariate dispersions among groups we used the computer program PERMDISP, which calculates distances from observations to their centroids and compares the average of these distances among groups, using permutation-based ANOVA (Anderson 2004).

The relationship between invertebrate abundance or diversity and urchin density (prediction 1, above) was tested using nonparametric correlation (Kendall's tau), since the data did not conform to linearity and homoscedasticity assumptions for ordinary least squares regression. Relationships between vertical and hori- 
Table 1. List of sessile invertebrate taxa by site (for site abbreviations see Fig. 1) and substrate orientation $(\mathrm{H}=$ horizontal, $\mathrm{V}=$ vertical)

\begin{tabular}{|c|c|c|c|c|c|c|c|c|c|c|c|c|c|c|c|c|}
\hline \multirow[t]{2}{*}{ Taxa } & \multicolumn{2}{|c|}{ SR } & \multicolumn{2}{|c|}{ WI } & \multicolumn{2}{|c|}{$\mathrm{TC}$} & \multicolumn{2}{|c|}{$\mathrm{BP}$} & \multicolumn{2}{|c|}{$\mathrm{MC}$} & \multicolumn{2}{|c|}{ CR } & \multicolumn{2}{|c|}{ WC } & \multicolumn{2}{|c|}{ PU } \\
\hline & $\mathrm{H}$ & $\mathrm{V}$ & $\mathrm{H}$ & $\mathrm{V}$ & $\mathrm{H}$ & V & $\mathrm{H}$ & $\mathrm{V}$ & $\mathrm{H}$ & V & $\mathrm{H}$ & $\mathrm{V}$ & $\mathrm{H}$ & $\mathrm{V}$ & $\mathrm{H}$ & $\mathrm{V}$ \\
\hline \multicolumn{17}{|l|}{ Ascidiacea } \\
\hline Aplidium glabrum & & $\mathrm{x}$ & & $\mathrm{x}$ & & $\mathrm{x}$ & & $\mathrm{x}$ & & $\mathrm{x}$ & & $\mathrm{x}$ & & $\mathrm{x}$ & & $\mathrm{x}$ \\
\hline Dendrodoa carnea & $\mathrm{x}$ & $\mathrm{x}$ & & $\mathrm{x}$ & & $\mathrm{x}$ & $\mathrm{x}$ & $\mathrm{x}$ & & $\mathrm{x}$ & & & & $\mathrm{x}$ & $\mathrm{x}$ & $\mathrm{x}$ \\
\hline Molgula citrina & $\mathrm{x}$ & $\mathrm{x}$ & & $\mathrm{x}$ & $\mathrm{x}$ & $\mathrm{x}$ & $\mathrm{x}$ & $\mathrm{x}$ & & $\mathrm{x}$ & $\mathrm{x}$ & & $\mathrm{x}$ & $\mathrm{x}$ & $\mathrm{x}$ & $\mathrm{x}$ \\
\hline Molgula sp. & & & & & $\mathrm{x}$ & & & $\mathrm{x}$ & & & & $\mathrm{x}$ & & $\mathrm{x}$ & & $\mathrm{x}$ \\
\hline Boltenia echinata L. & & $\mathrm{x}$ & & & & & & $\mathrm{x}$ & & & & $\mathrm{x}$ & & $\mathrm{x}$ & & $\mathrm{x}$ \\
\hline Boltenia ovifera L. & & & & & & & & & & $\mathrm{x}$ & & $\mathrm{x}$ & & & & $\mathrm{x}$ \\
\hline Didemnum albidum & $\mathrm{x}$ & $\mathrm{x}$ & & $\mathrm{x}$ & & $\mathrm{x}$ & & $\mathrm{x}$ & $\mathrm{x}$ & $\mathrm{x}$ & $\mathrm{x}$ & $\mathrm{x}$ & & $\mathrm{x}$ & & $\mathrm{x}$ \\
\hline Botryllus schlosseri Pallas & & $\mathrm{x}$ & & & & $\mathrm{x}$ & & & & & & & & & & \\
\hline Botrylloides violaceus & $\mathrm{x}$ & $\mathrm{x}$ & $\mathrm{x}$ & $\mathrm{x}$ & $\mathrm{x}$ & $\mathrm{x}$ & & & & & & & & & & \\
\hline Halocynthis pyriformis Rathke & & $\mathrm{x}$ & & & & & & & & $\mathrm{x}$ & & $\mathrm{x}$ & & & & $\mathrm{x}$ \\
\hline Ascidiella aspersa Müller & & $\mathrm{x}$ & & & & & & & & & & & & & & \\
\hline Didemnum vexillum & & & & & $\mathrm{x}$ & $\mathrm{x}$ & & & & & & & & & & \\
\hline Diplosoma sp. & & & & & $\mathrm{x}$ & $\mathrm{x}$ & & & & & & & & & & \\
\hline Trididemnum sp. & & & & & & & & $\mathrm{x}$ & & & & & & $\mathrm{x}$ & & \\
\hline \multicolumn{17}{|l|}{ Porifera } \\
\hline Leucoselenia sp. & & $\mathrm{x}$ & & & & & & & & & & & & $\mathrm{x}$ & & $\mathrm{x}$ \\
\hline Halichondria sp. & & $\mathrm{x}$ & & $\mathrm{x}$ & & & & & & $\mathrm{x}$ & & $\mathrm{x}$ & & $\mathrm{x}$ & & $\mathrm{x}$ \\
\hline Halisarca sp. & $\mathrm{x}$ & $\mathrm{x}$ & & $\mathrm{x}$ & & & & $\mathrm{x}$ & & & & $\mathrm{x}$ & & $\mathrm{x}$ & & $\mathrm{x}$ \\
\hline Myxilla fimbriata Bowerbank & & $\mathrm{x}$ & & & & & & $\mathrm{x}$ & & & & $\mathrm{x}$ & & & & $\mathrm{x}$ \\
\hline Isodictya palmata & $\mathrm{x}$ & & & & & $\mathrm{x}$ & & & & $\mathrm{x}$ & & $\mathrm{x}$ & $\mathrm{x}$ & $\mathrm{x}$ & $\mathrm{x}$ & \\
\hline Hymedesmia & & & & $\mathrm{x}$ & & & & & & & $\mathrm{x}$ & & $\mathrm{x}$ & $\mathrm{x}$ & & $\mathrm{x}$ \\
\hline Haliclona permollis Bowerbank & & & & & & & & $\mathrm{x}$ & & $\mathrm{x}$ & & & & & & \\
\hline Haliclona oculata Pallas & & & & & & & & $\mathrm{x}$ & & & & & & & & \\
\hline Scypha sp. & & & & & & & & & & $\mathrm{x}$ & & $\mathrm{x}$ & & & & $\mathrm{x}$ \\
\hline Iophon nigricans Bowerbank & & & & & & & & & & & & $\mathrm{x}$ & & $\mathrm{x}$ & & $\mathrm{x}$ \\
\hline Cliona celata Grant & & & & & & & & & & & & & & $\mathrm{x}$ & & $\mathrm{x}$ \\
\hline Plocamionida ambigua Bowerbank & & & & & & & & & & & & & & & & $\mathrm{x}$ \\
\hline \multicolumn{17}{|l|}{ Bryozoa } \\
\hline Schizomavella auriculata Canu & $\mathrm{x}$ & $\mathrm{x}$ & & $\mathrm{x}$ & & & & $\mathrm{x}$ & & $\mathrm{x}$ & & $\mathrm{x}$ & & $\mathrm{x}$ & & $\mathrm{x}$ \\
\hline Parasmittina jeffreysi Norman & & & & & & & & & & $\mathrm{x}$ & & $\mathrm{x}$ & & $\mathrm{x}$ & & \\
\hline Bugula turrida Desor & & $\mathrm{x}$ & & & & & & & & & & $\mathrm{x}$ & & & & \\
\hline Dendrobaenia murrayana Bean & & $\mathrm{x}$ & & $\mathrm{x}$ & & $\mathrm{x}$ & & & & $\mathrm{x}$ & & $\mathrm{x}$ & & $\mathrm{x}$ & & $\mathrm{x}$ \\
\hline Crisia sp. & & $\mathrm{x}$ & & $\mathrm{x}$ & & & & $\mathrm{x}$ & & $\mathrm{x}$ & & & & $\mathrm{x}$ & & $\mathrm{x}$ \\
\hline Caberia ellisii Fleming & & $\mathrm{x}$ & & $\mathrm{x}$ & & & & & & $\mathrm{x}$ & & $\mathrm{x}$ & & $\mathrm{x}$ & & $\mathrm{x}$ \\
\hline Tubulipora sp. & & & & & & & & & & & & $\mathrm{x}$ & & & & \\
\hline Membranipora membranacea L. & & & & & & & & & & & & & & $\mathrm{x}$ & & \\
\hline Cnidaria & & & & & & & & & & & & & & & & \\
\hline Alcyonium siderium Verrill & $\mathrm{x}$ & $\mathrm{x}$ & & & & & & & & & & & & & & \\
\hline Urticina crassicornis Müller & & & & & & & & & $\mathrm{x}$ & $\mathrm{x}$ & $\mathrm{x}$ & $\mathrm{x}$ & & & & $\mathrm{x}$ \\
\hline Metridium senile L. & & $\mathrm{x}$ & & & & $\mathrm{x}$ & & & & & $\mathrm{x}$ & & & $\mathrm{x}$ & $\mathrm{x}$ & $\mathrm{x}$ \\
\hline Fagesia lineata Verrill & & $\mathrm{x}$ & & & & & & & & & & & & & & \\
\hline Halecium sp. & & $\mathrm{x}$ & & & & & & & & & & & & $\mathrm{x}$ & & \\
\hline Tubularia sp. & $\mathrm{x}$ & $\mathrm{x}$ & & & & $\mathrm{x}$ & & & & & $\mathrm{x}$ & & & & & \\
\hline Obelia sp. & & & & & & & & & $\mathrm{x}$ & $\mathrm{x}$ & & $\mathrm{x}$ & & $\mathrm{x}$ & & \\
\hline Other & & & & & & & & & & & & & & & & \\
\hline Anomia sp & & $\mathrm{x}$ & & & & & & & & $\mathrm{x}$ & $\mathrm{x}$ & $\mathrm{x}$ & & & $\mathrm{x}$ & $\mathrm{x}$ \\
\hline Modiolus modiolus & $\mathrm{x}$ & $\mathrm{x}$ & $\mathrm{x}$ & $\mathrm{x}$ & & $\mathrm{x}$ & & $\mathrm{x}$ & $\mathrm{x}$ & & & & & $\mathrm{x}$ & & \\
\hline Mytilus sp. & & & & & & & & & $\mathrm{x}$ & $\mathrm{x}$ & & $\mathrm{x}$ & & $\mathrm{x}$ & & $\mathrm{x}$ \\
\hline Balanus balanus da Costa & & $\mathrm{x}$ & & $\mathrm{x}$ & $\mathrm{x}$ & $\mathrm{x}$ & & $\mathrm{x}$ & $\mathrm{x}$ & $\mathrm{x}$ & & $\mathrm{x}$ & & $\mathrm{x}$ & & \\
\hline Semibalanus balanoides L. & $\mathrm{x}$ & $\mathrm{x}$ & & $\mathrm{x}$ & & & & & & $\mathrm{x}$ & & & & $\mathrm{x}$ & & \\
\hline Spirorbis sp. & $\mathrm{x}$ & $\mathrm{x}$ & $\mathrm{x}$ & $\mathrm{x}$ & & $\mathrm{x}$ & $\mathrm{x}$ & $\mathrm{x}$ & $\mathrm{x}$ & $\mathrm{x}$ & & $\mathrm{x}$ & & $\mathrm{x}$ & $\mathrm{x}$ & $\mathrm{x}$ \\
\hline Myxicola & & $\mathrm{x}$ & & & & $\mathrm{x}$ & & $\mathrm{x}$ & & $\mathrm{x}$ & & & & & & $\mathrm{x}$ \\
\hline Filograna implexa Berkeley & & & & & & & & $\mathrm{x}$ & & $\mathrm{x}$ & & $\mathrm{x}$ & & $\mathrm{x}$ & & \\
\hline Terebratulina septentrionalis Couthouy & & & & & & & & & & $\mathrm{x}$ & & $\mathrm{x}$ & & $\mathrm{x}$ & & $\mathrm{x}$ \\
\hline
\end{tabular}


zontal sessile invertebrate species richness among sites, and species richness versus percent cover, were explored using least squares regression. Plots of residual versus predicted values were examined to evaluate possible violations of regression assumptions. Grazer densities and percent cover of major space occupiers were analyzed using 2-way ANOVA, with site as a random factor and orientation (vertical vs. horizontal) a fixed factor. Proportion cover data were transformed to logits, calculated as $\ln [\mathrm{p} /(\mathrm{p}-1)]$ (Ramsey \& Schafer 2002), and abundance data were log transformed to homogenize variances for analysis. F-tests were used for planned multiple comparisons of means. The above analyses were carried out using JMP software (v. 5.1 for the Macintosh, SAS Institute).

\section{RESULTS}

\section{Invertebrate species richness}

\section{Local richness}

Fifty species were identified in a total of 288 photographs covering $72 \mathrm{~m}^{2}$ (Table 1). Vertical walls had, on average, 4 times more sessile invertebrate species than horizontal rock surfaces (Fig. 2), with species richness ranging from 15 to 31 species (mean $24.8 \pm$ $0.1 \mathrm{SE}, \mathrm{n}=8$ ) on vertical walls compared to 3 to 12 species on horizontal rock (mean $6.0 \pm 0.3 \mathrm{SE}, \mathrm{n}=8$ ). This difference was highly significant whether richness was estimated with $S_{\text {obs }}$ or Chao2 (ANOVA, p < 0.001, Table 2). Richness on horizontal surfaces was not significantly related to that on vertical surfaces across sites $\left(F_{1,6}=1.39, \mathrm{p}=0.3\right)$. Species richness of sessile invertebrates on vertical walls varied parabolically with invertebrate percent cover, such that richness declined when open space was limited (Fig. 3A). The decline in diversity at high percent cover reflects the dominance of the (likely invasive) compound ascidian Didemnum vexillum Kott at one site, Thrumcap (the only site where this species was present). When Thrumcap was removed, richness was linearly related to percent cover (Fig. 3B). However, in both cases, percent cover explained less than half of the variation in
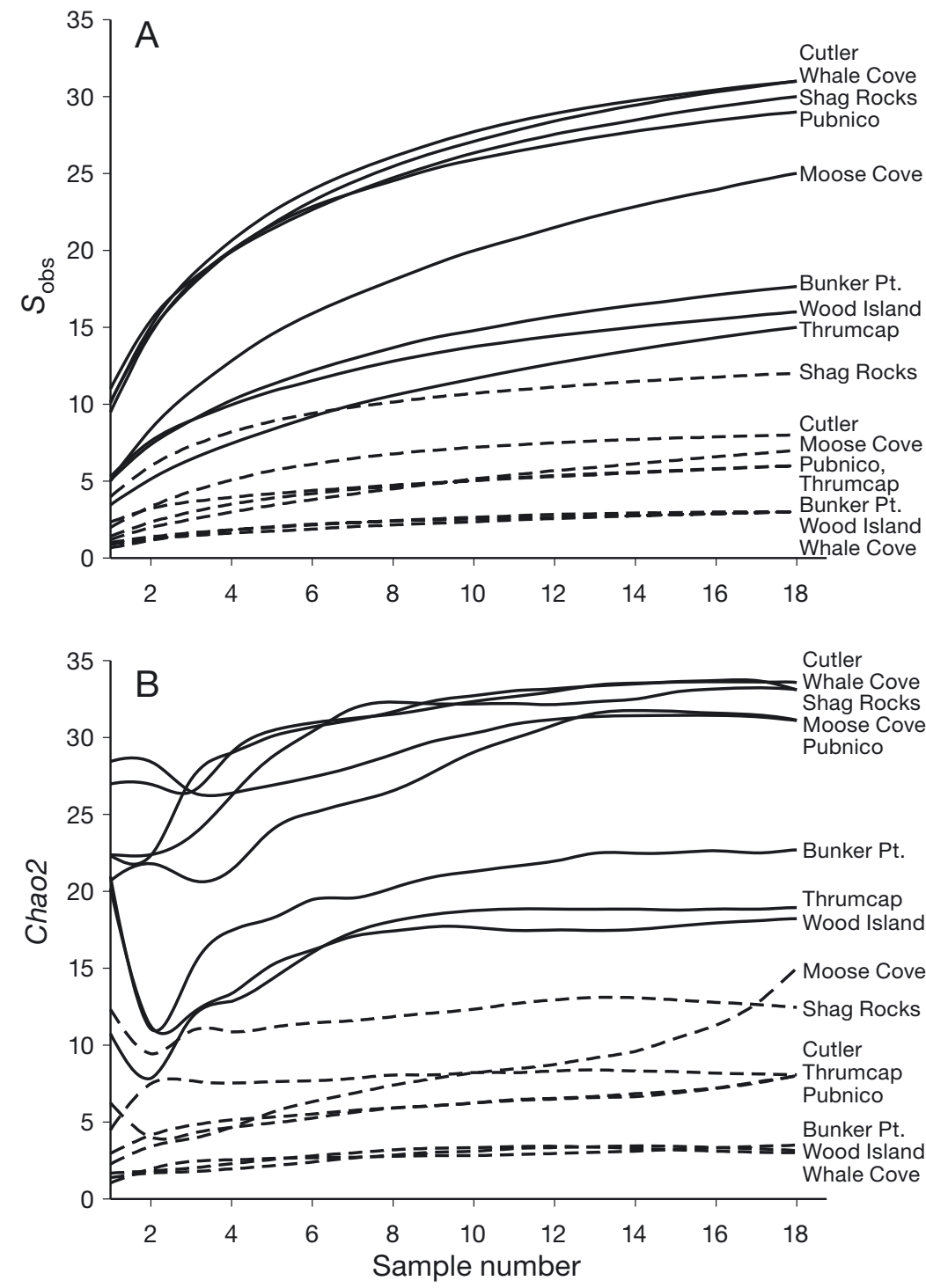

Fig. 2. Species accumulation curves showing (A) $S_{\text {obs }}$ and (B) Chao2 (see 'Materials and methods: Statistical analysis') for sessile invertebrates on vertical (solid line) and horizontal (dashed line) rock surfaces at 8 sites in the Gulf of Maine. Curves were generated with EstimateS software (Colwell 1997) using means of 1000 random draws of the number of quadrats sampled from a total of 18 at each point, so curves do not depend on sampling order

Table 2. ANOVA analyses of species richness of sessile invertebrates, estimated by $C h a o 2$ and $S_{\text {obs, }}$ by the fixed factor orientation (horizontal vs. vertical)

\begin{tabular}{|lcccc|}
\hline Source & df & MS & $F$ & $p$ \\
\hline Chao2 & & & & \\
$\begin{array}{l}\text { Orientation } \\
\text { Residual }\end{array}$ & 1 & 1615.0 & 50.83 & $<0.0001$ \\
$\boldsymbol{S}_{\text {obs }}$ & 14 & 31.8 & & \\
$\begin{array}{l}\text { Orientation } \\
\text { Residual }\end{array}$ & 1 & 1344.1 & 45.63 & $<0.0001$ \\
\hline
\end{tabular}




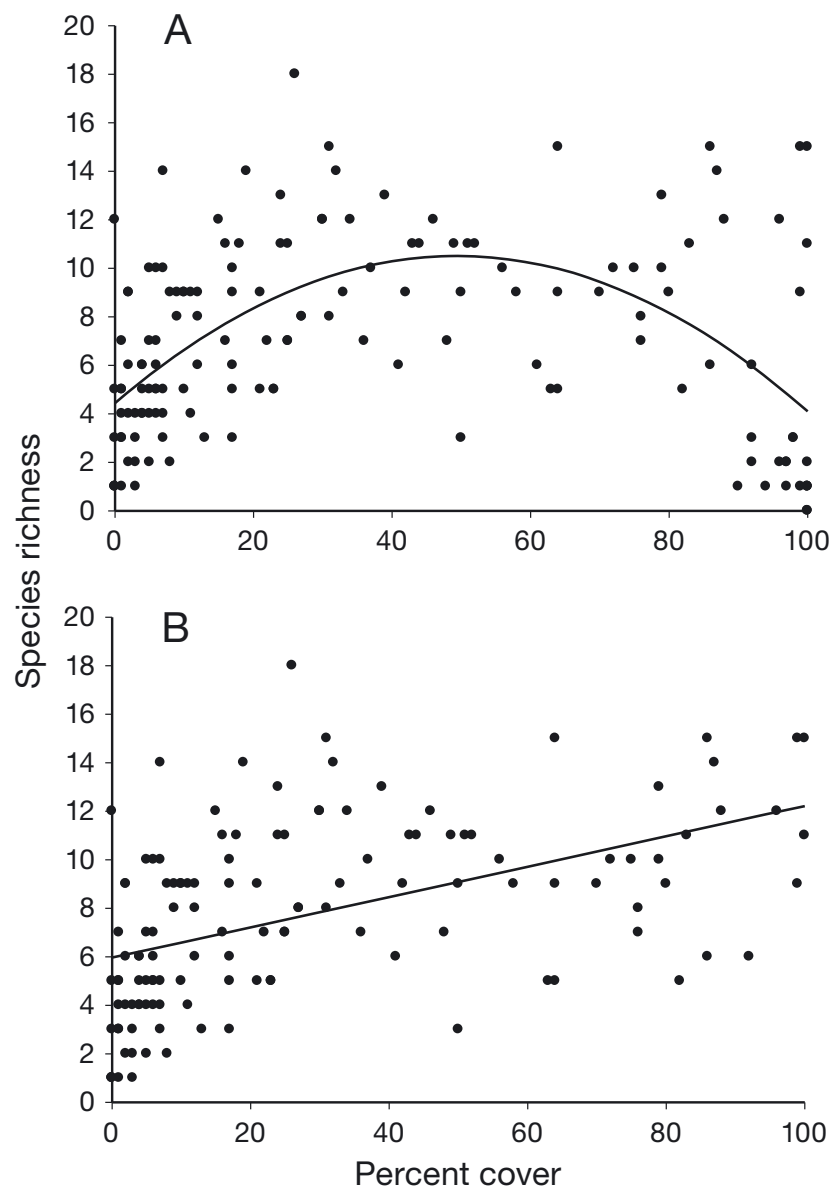

Fig. 3. Sessile invertebrate species richness vs. percent cover on vertical walls; each point represents a quadrat. (A) All sites, fitted with the second-order polynomial (quadratic) equation: richness $=7.9+0.06 \times \%$ cover $-0.002 \times(\%$ cover $37.5)^{2}\left(\mathrm{r}^{2}=0.31, \mathrm{p}<0.0001\right)$. (B) All sites excluding Thrumcap, fitting the linear regression: richness $=5.97+0.06 \times \%$ cover $\left(\mathrm{r}^{2}=0.42, \mathrm{p}<0.0001\right)$

species richness. Observed species richness of horizontal quadrats at Shag Rocks was exactly identical before and after algal removal, indicating that the photographic method used was a good estimator of richness.

\section{Beta diversity}

The number of species shared $\left(S_{\text {obs, }}\right.$ Bray-Curtis, or Chao2 estimated) between sites was unrelated to the geographic distances separating the sites for both horizontal and vertical surfaces (e.g. vertical Chao: Mantel statistic $\mathrm{r}=-0.09, \mathrm{p}=0.6$; horizontal Chao: Mantel statistic $r=0.25, p=0.2$ ). Species composition differed little throughout the GOM, with the most distant sites being similar to adjacent sites (Fig. 4). The average dissimilarity of vertical surfaces between sites was higher than that

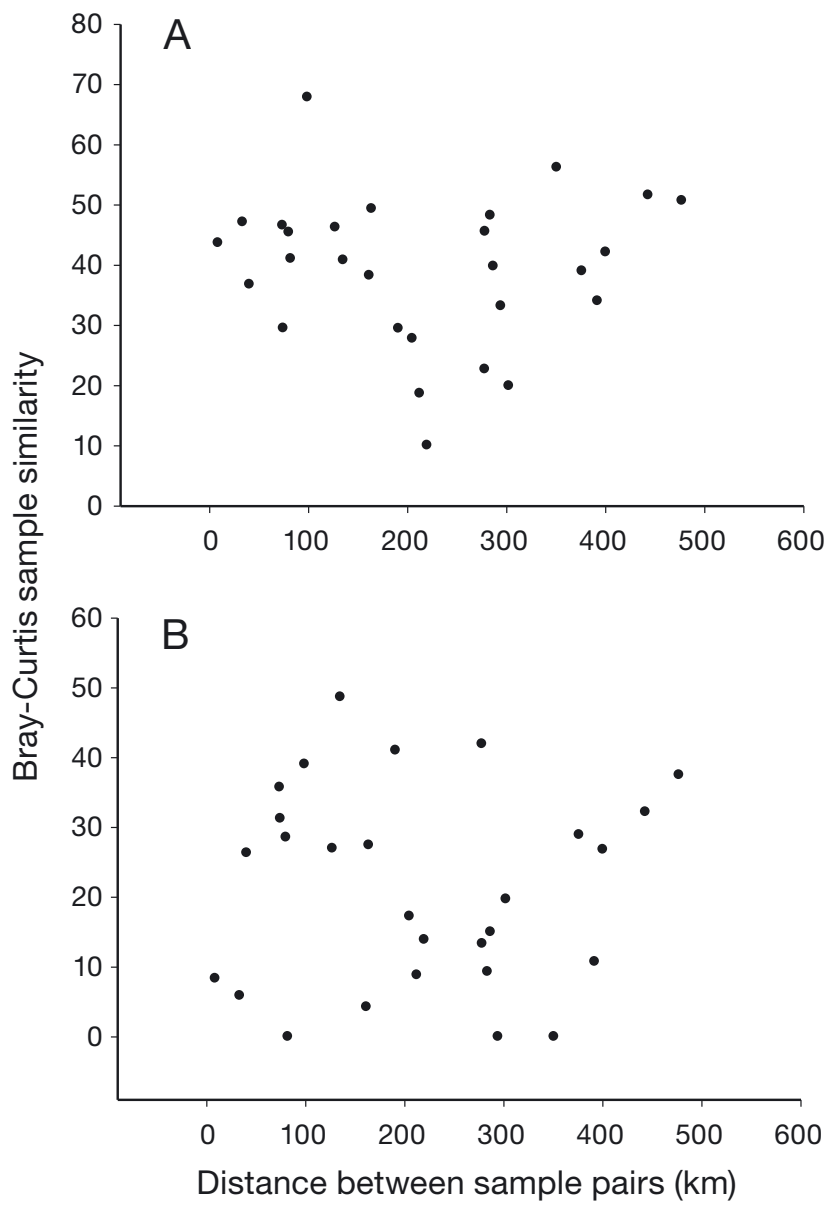

Fig. 4. Bray-Curtis sample similarity index versus distance between all site pairs for (A) vertical surfaces and (B) horizontal surfaces

of horizontal sites (mean Bray-Curtis distance 39.3 \pm 3 SE for vertical walls, $21.4 \pm 3 \mathrm{SE}$ for horizontal platforms). Dissimilarity among quadrats within sites was greater than that between different sites (mean betweenquadrat Bray-Curtis dissimilarity distance 50.2 $\pm 3 \mathrm{SE}$ for vertical walls, $58.1 \pm 5 \mathrm{SE}$ for horizontal platforms).

\section{Abundance}

Abundance of space occupiers

There was a significant interaction between site and substrate orientation on invertebrate cover (Table 3). Percentage cover of invertebrates, however, was significantly higher on vertical compared to horizontal surfaces at every site (multiple comparison F-tests, p < 0.0001, Fig. 5), with only the magnitude of the difference varying between sites. There was no evidence that the algal canopy on horizontal surfaces significantly affected estimates of invertebrate cover where this was tested at 
Table 3. ANOVA comparing the proportional cover of selected categories between orientations (fixed: horizontal vs. vertical) at 8 sites (random). Data were logit transformed, which homogenized variances for most categories (Levene's test, $\mathrm{p}>$ 0.05). Significant values are in bold. Inequality signs indicate the direction of significant differences of means between $(\mathrm{H})$ horizontal and (V) vertical substrates at the named sites. See 'Results: Abundance' for results of multiple comparisons done using F-tests

\begin{tabular}{|c|c|c|c|c|}
\hline Source & df & MS & $F$ & $\mathrm{p}$ \\
\hline \multicolumn{5}{|c|}{ Invertebrates (excluding mussels) ${ }^{a}$} \\
\hline Orientation & 1 & 1854.9 & 90.98 & $<0.0001$ \\
\hline Site & 7 & 266.5 & 42.98 & $<0.0001$ \\
\hline Orientation $\times$ Site & 7 & 20.4 & 3.26 & $<0.01$ \\
\hline Residual & 272 & 6.2 & & \\
\hline \multicolumn{5}{|l|}{ Macroalgae } \\
\hline Orientation & 1 & 1020.0 & 11.22 & 0.01 \\
\hline Site & 7 & 359.2 & 35.22 & $<0.0001$ \\
\hline Orientation $\times$ Site & 7 & 90.9 & 8.92 & $<0.0001$ \\
\hline Residual & 272 & 10.2 & & \\
\hline \multicolumn{5}{|l|}{ Peysonnalia sp. } \\
\hline Orientation & 1 & 8.9 & 0.31 & 0.600 \\
\hline Site & 7 & 209.6 & 38.81 & $<0.0001$ \\
\hline Orientation $\times$ Site & 7 & 28.7 & 5.32 & $<0.0001$ \\
\hline Residual & 272 & 5.4 & & \\
\hline \multicolumn{5}{|l|}{ Mussels } \\
\hline Orientation & 1 & 0.4 & 0.12 & 0.7 \\
\hline Site & 7 & 80.9 & 20.74 & $<0.0001$ \\
\hline Orientation $\times$ Site & 7 & 3.5 & 0.89 & 0.5 \\
\hline Residual & 272 & 3.9 & & \\
\hline \multicolumn{5}{|c|}{ Crustose coralline algae } \\
\hline Orientation & 1 & 146.9 & 2.46 & 0.2 \\
\hline Site & 7 & 337.3 & 51.89 & $<0.0001$ \\
\hline Orientation $\times$ Site & 7 & 59.7 & 9.24 & $<0.0001$ \\
\hline Residual & 272 & 6.5 & & \\
\hline \multicolumn{5}{|l|}{ Tube complex ${ }^{a}$} \\
\hline Orientation & 1 & 100.0 & 0.72 & 0.4 \\
\hline Site & 7 & 112.5 & 15.41 & $<0.0001$ \\
\hline Orientation $\times$ Site & 7 & 139.0 & 19.10 & $<0.0001$ \\
\hline Residual & 272 & 7.3 & & \\
\hline
\end{tabular}

the Shag Rocks site ( $t$-test: $\mathrm{df}=18, \mathrm{p}=0.4)$. Macroalgae was significantly more abundant on horizontal surfaces at all sites except Moose Cove and Cutler, which had low algal cover overall and very high urchin densities (multiple comparison F-tests, p < 0.0001, Figs. 5 \& 6). Crustose coralline algae, Peysonnalia spp. and tube complex did not differ between vertical and horizontal platforms (multiple comparison F-tests, p < 0.0001, Table 3, Fig. 5). Mussel abundance also did not differ with substrate orientation ( $p=0.74$, Table 3 ). Percent cover of sessile invertebrates was negatively related, albeit weakly, to sea urchin density on horizontal (Kendall's tau $=-0.39$, p < 0.001 ) and vertical (Kendall's tau $=-0.24, \mathrm{p}<0.001$ ) substrate. On vertical walls, this correlation was driven by the anomalous site Thrumcap, which had high invertebrate cover and low urchin density (Figs. 5 \& 6). There
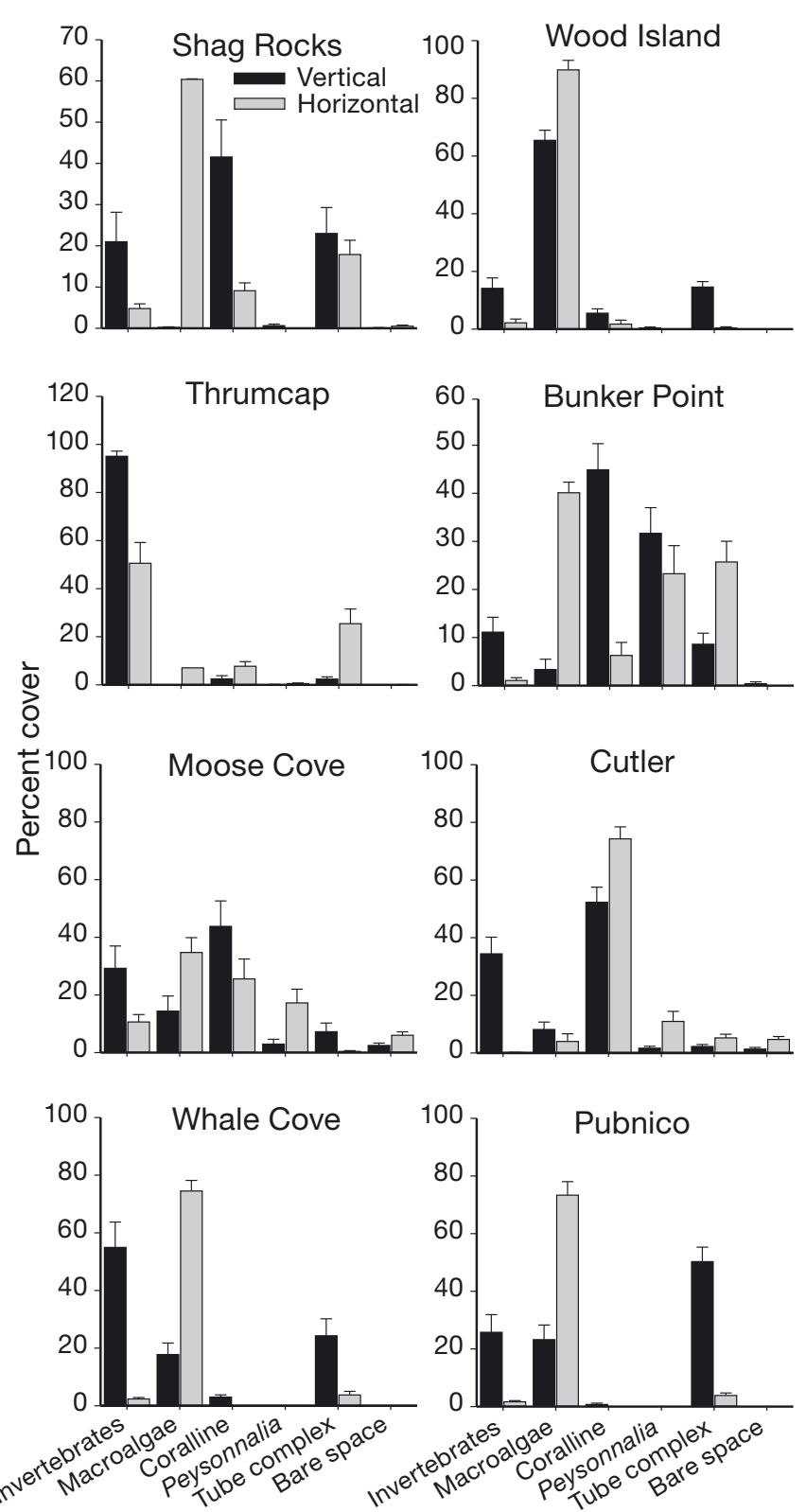

Fig. 5. Percent cover of 6 categories: invertebrates, macroalgae, crustose coralline algae, Peysonnalia sp., tube complex, and bare space, for vertical and horizontal rock substrate at the 8 sites. Error bars are $1 \mathrm{SE}$

was no correlation with Thrumcap removed (Kendall's tau $=0.08, p=0.20$ ). Invertebrate richness was positively correlated with urchin density on vertical walls (Kendall's tau $=0.16, \mathrm{p}=0.01$ ) but not on horizontal platforms (Kendall's tau $=0.07, \mathrm{p}=0.30$ ). This weak positive correlation was again driven by the anomalous site Thrumcap, which had low richness due to high abundance of Didemnum vexillum, and low urchin density (Fig. 6). With Thrumcap removed, the correlation disappeared (Kendall's tau $=0.003, \mathrm{p}=0.95$ ). 

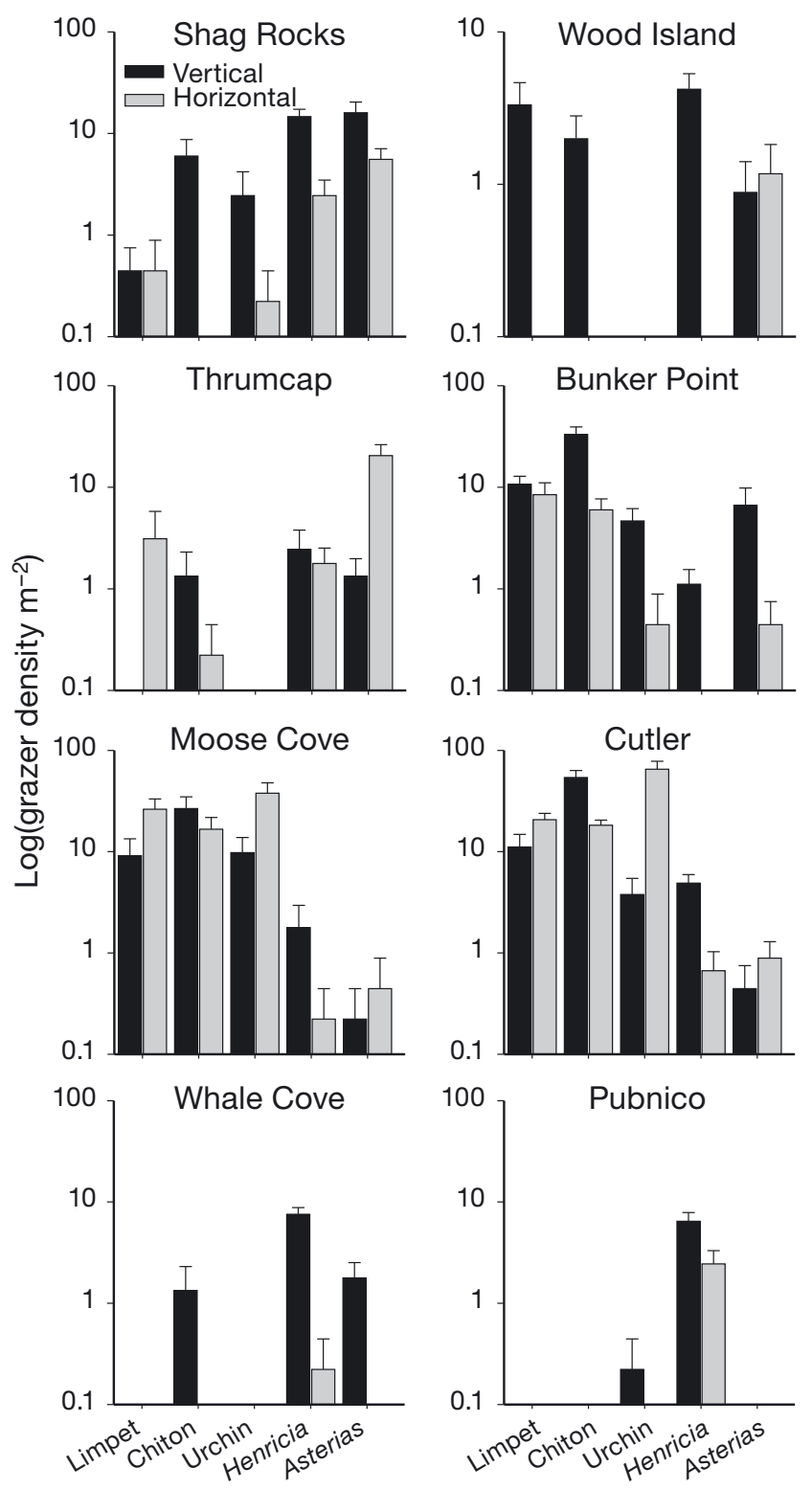

Fig. 6. Grazer densities $\left(\mathrm{m}^{-2}\right)$ on a log scale at the 8 sites, by substrate orientation. Error bars are $1 \mathrm{SE}$

\section{Community structure}

Most invertebrate species (27 out of 50) were found only on vertical walls (Table 2). All Bryozoa, 7 out of 12 species of Porifera, and 7 out of 14 tunicate species were confined to walls. Only 2 out 7 cnidarian species were confined to walls. No sessile invertebrate species was found only on horizontal substrate. NMDS ordination of invertebrate species showed a general separation of horizontal and vertical communities (Fig. 7A). CAP analysis indicated that assemblages on vertical and horizontal substrates were significantly different
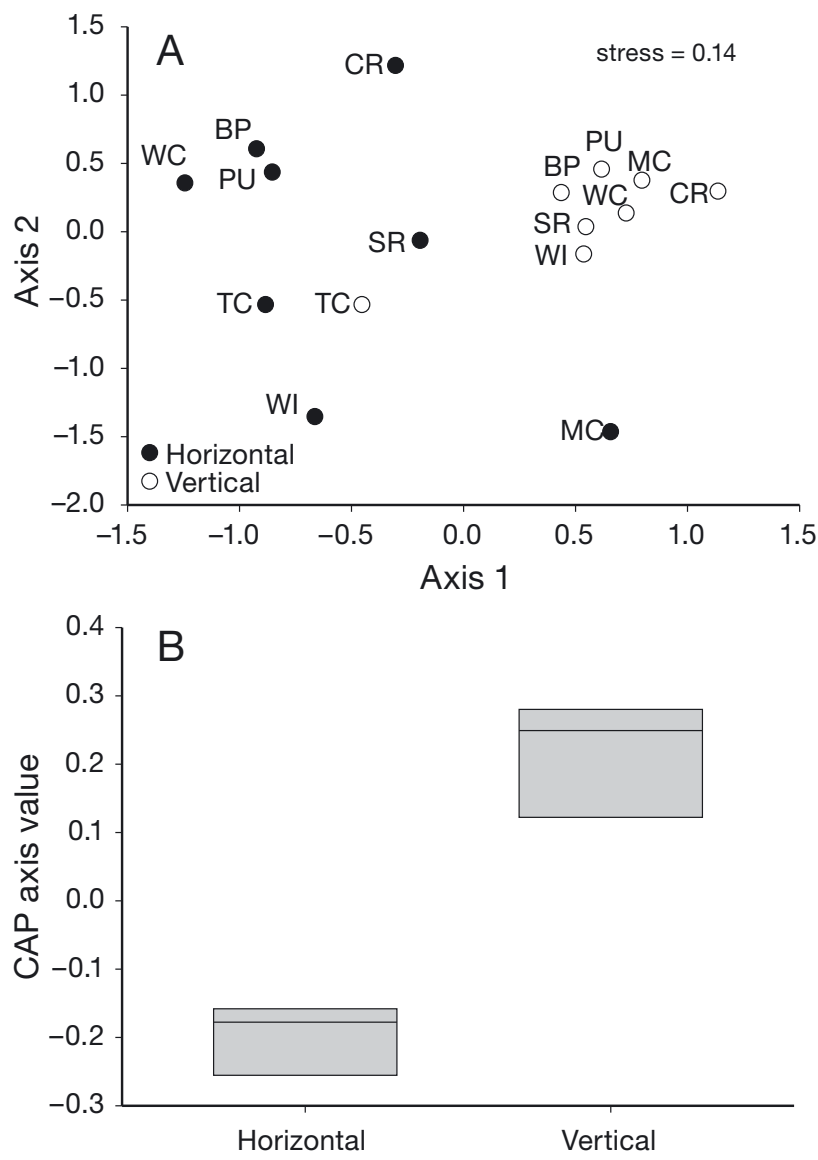

Fig. 7. (A) NMDS ordination plot of sites separated by orientation. For site abbreviations see Fig. 1. (B) CAP axis values for vertical and horizontal areas. Boxes represent upper and lower quartiles divided by a line representing the median

(Fig. 7B, $\mathrm{p}=0.0008$ from 9999 permutations). CAP axis values for vertical surfaces averaged 0.2 , while horizontal surfaces averaged -0.2 ; therefore, positive correlation coefficients with the original species data separated vertical plots while negative correlations were associated with horizontal plots. Many native invertebrate species were positively correlated with the CAP axis values, particularly the colonial tunicate Didemnum albidum Verrill and the tubeworm Spirorbis sp. (Fig. 8). Negatively correlated species that strongly influenced horizontal plots were dominated by invasive colonial tunicates (Didemnum vexillum, Diplosoma sp., and Botrylloides violaceus Oka), and by the sponge Isodictya palmata Ellis \& Solander (Fig. 8). Leave-one-out analysis of the groups (vertical and horizontal) showed high classification accuracy: all siteorientation combinations were correctly classified as vertical or horizontal based on the species present except Thrumcap vertical, which was incorrectly classified as horizontal. This site was heavily dominated by 


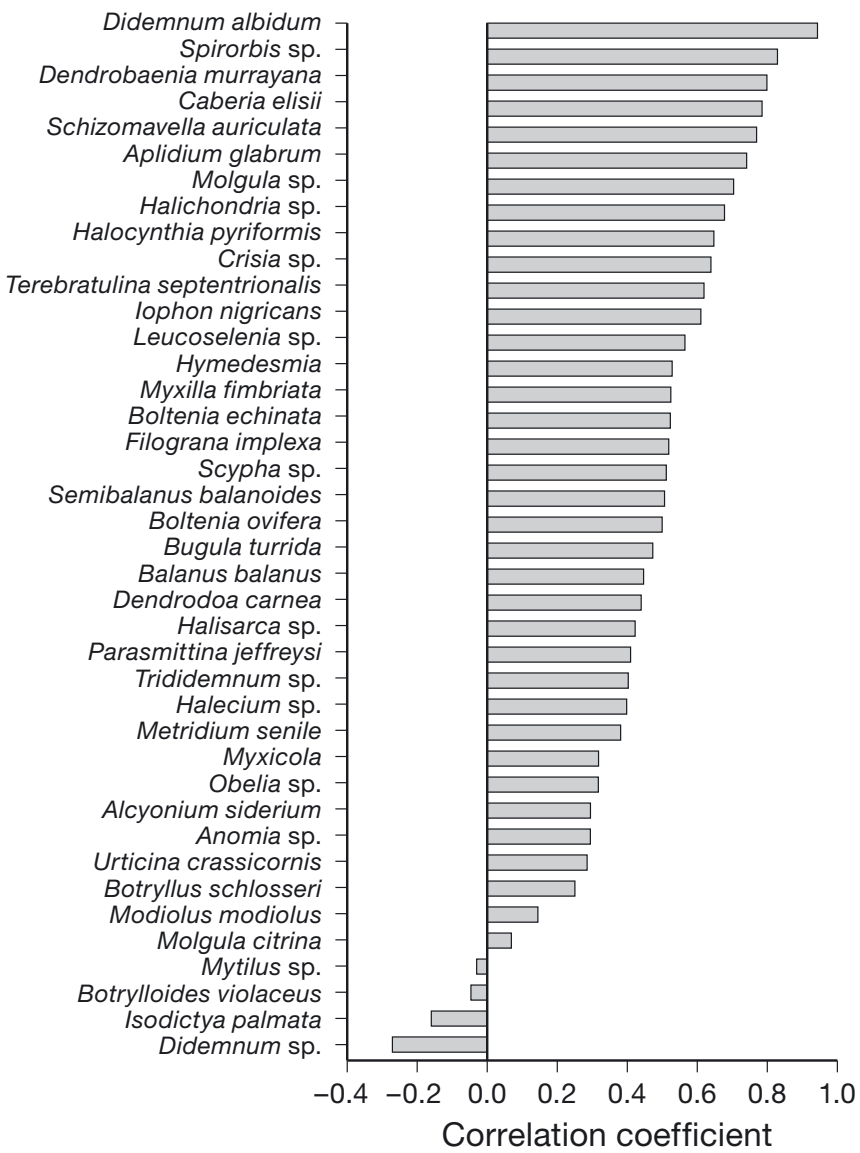

Fig. 8. Correlation coefficients for species influencing CAP axis values. Species occurring less than 6 times were omitted. Species with low and negative values more strongly influenced horizontal surfaces, while species with positive values strongly influenced vertical surfaces

D. vexillum on both the horizontal and vertical substrates. Species composition of horizontal surfaces was significantly more variable (sites were more dispersed, Fig. 7A) compared to vertical surfaces (PERMDISP, 9999 permutations, $F_{1,15}=6.88, \mathrm{p}=0.02$ ).

\section{Grazer abundance}

Density of Strongylocentrotus droebachiensis did not differ with substrate angle (Table 4, Fig. 6), although at the sites with very high urchin densities, Moose Cove and Cutler, urchins were much more abundant on horizontal substrate (Fig. 6). Site and orientation interacted to affect densities of all grazers (Table 4). Chitons and the asteroid Henricia sanguinolenta were the only grazers with patterns of abundance corresponding to substrate angle: both were more abundant on vertical walls at sites where they were common (Table 4, Fig. 6).

\section{DISCUSSION}

Vertical rock walls in the shallow subtidal Gulf of Maine have both greater abundance and greater species richness of sessile suspension-feeding invertebrates compared to horizontal rock surfaces. This is consistent with previous work at local sites in the GOM where communities of sessile suspension feeders dominated vertical or undercut rock surfaces (Witman \& Cooper 1983, Sebens 1986a). The lack of a relationship between vertical and horizontal invertebrate species richness suggests that most larvae recruiting on horizontal rock do not survive, or that larvae from local rock walls avoid horizontal surfaces. Graham \& Sebens (1996) found that the abundance of sessile invertebrate larvae decreased with distance away from rock walls at Shag Rocks. Poor post-recruitment survival on nearby horizontal surfaces is supported by the experimental results of Miller \& Etter (2008), who demonstrated that invertebrate larvae settled on horizontal surfaces near walls, whether these surfaces were shaded or unshaded, but survived better on shaded surfaces. Larval behaviors such as negative phototropism, therefore, may be adaptations to facilitate recruitment to successful parental habitats, rather than selection of new habitats (Miller \& Etter 2008).

The significant interaction between site and orientation (Table 3) reflects the wide variation in the magnitude of the difference between horizontal and vertical surfaces across sites (Fig. 4). The most anomalous site was Thrumcap, where high invertebrate cover on the horizontal was almost entirely composed of 2 colonial ascidians, Didemnum vexillum and Botrylloides violaceus. The latter invaded the GOM around the 1970s (Berman et al. 1992, listed incorrectly as B. diegensis Ritter \& Forsyth). D. vexillum is likely an invader from the northwestern Pacific (Stefaniak et al. 2009, Lambert 2009, Bullard et al. 2007), and has been locally abundant around that site since at least 1988 (P. Yund pers. comm.). Both species grow rapidly (R. Miller unpubl. data), often overgrowing other species (Berman et al. 1992, Bullard et al. 2007), and B. violaceus was unpalatable to urchins in feeding trials (Simoncini \& Miller 2007). The presence of these competitively dominant species, particularly $D$. vexillum, which often occupied $100 \%$ of a quadrat, caused a hump-shaped relationship between percent cover and species richness (Fig. 3A). A strong negative influence of a single competitively dominant species on diversity has been found at rock wall sites in South Africa and Australia (Witman et al. 2004), suggesting that this may be a general phenomenon in sessile invertebrate communities. High abundance of competitive dominants can result from low disturbance frequency, the left tail of the familiar intermediate disturbance relationship (Parvi- 
Table 4. Analysis of variance comparing grazer density $\left(\mathrm{m}^{-2}\right)$ among orientations (fixed: horizontal, vertical) at 8 sites (random). Log transformations were done to equalize variances (Levene's test, $p>0.05$ ). Significant $p$-values are in bold. $\mathrm{MC}=$ multiple comparisons done using F-tests. Inequality signs indicate the direction of significant differences of means between $(\mathrm{H})$ horizontal and (V) vertical substrates at the named sites (for site abbreviations see Fig. 1). ${ }^{*} \mathrm{p}<0.05,{ }^{* *} \mathrm{p}<0.01,{ }^{* * *} \mathrm{p}<0.001$

\begin{tabular}{|c|c|c|c|c|c|}
\hline Source & $\mathrm{df}$ & MS & $F$ & $\mathrm{p}$ & $\mathrm{MC}$ \\
\hline \multicolumn{6}{|l|}{ Chitons } \\
\hline Orientation & 1 & 49.6 & 11.19 & 0.01 & $\mathrm{PO}, \mathrm{TC}, \mathrm{CR}, \mathrm{WC}: \mathrm{V}=\mathrm{H}$; \\
\hline Site & 7 & 97.4 & 57.29 & $<0.0001$ & $\mathrm{MC}: \mathrm{V}>\mathrm{H}^{*}$ \\
\hline Orientation $\times$ Site & 7 & 4.4 & 2.61 & 0.01 & $\mathrm{BF}: \mathrm{V}>\mathrm{H}^{* *}$ \\
\hline Residual & 272 & 1.7 & & & BP, SR: V > H*** \\
\hline \multicolumn{6}{|c|}{ Strongylocentrotus droebachiensis } \\
\hline Orientation & 1 & 3.9 & 0.15 & 0.71 & \\
\hline Site & 7 & 36.1 & 24.1 & $<0.0001$ & \\
\hline Orientation $\times$ Site & 7 & 25.9 & 17.25 & $<0.0001$ & \\
\hline Residual & 272 & 1.5 & & & \\
\hline \multicolumn{6}{|c|}{ Henricia sanguinolenta } \\
\hline Orientation & 1 & 177.2 & 16.31 & $<0.01$ & TC, MC: $V=H_{i}$ \\
\hline Site & 7 & 19.5 & 8.48 & $<0.0001$ & $\mathrm{BP}, \mathrm{PO}: \mathrm{V}>\mathrm{H}^{*}$ \\
\hline Orientation $\times$ Site & 7 & 10.9 & 4.83 & $<0.0001$ & all others: $\mathrm{V}>\mathrm{H}^{* * *}$ \\
\hline Residual & 272 & 2.3 & & & \\
\hline \multicolumn{6}{|l|}{ Tectura testudinalis } \\
\hline Orientation & 1 & 3.6 & 0.29 & 0.6 & \\
\hline Site & 7 & 64.5 & 35.8 & $<0.0001$ & \\
\hline Orientation $\times$ Site & 7 & 12.3 & 6.72 & $<0.001$ & \\
\hline Residual & 272 & 1.8 & & & \\
\hline \multicolumn{6}{|l|}{ Asterias spp. } \\
\hline Orientation & 1 & 4.0 & 0.34 & 0.6 & \\
\hline Site & 7 & 32.3 & 16.3 & $<0.0001$ & \\
\hline Orientation $\times$ Site & 7 & 11.8 & 5.96 & $<0.0001$ & \\
\hline Residual & 272 & 1.98 & & & \\
\hline
\end{tabular}

nen \& Meszéna 2009). Invasibility, conversely, is often facilitated by frequent or severe disturbance, or other processes that result in excess resources (Davis \& Pelsor 2001). Subtidal rock walls have low disturbance frequencies compared to horizontal platforms (Witman \& Dayton 2001). We have no reason to believe that Thrumcap has a disturbance regime that is different from the other sites, all of which were located off exposed rocky headlands. In this case, competitive dominance of these 2 species relative to native species, rather than excess resources created by disturbance, may be responsible for their success as invaders (Thompson 1991). This hypothesis deserves further examination, and may help explain why these species have invaded the subtidal, while most invasive ascidians are largely restricted to fouling habitats (Osman \& Whitlatch 2007).

Species composition of invertebrate communities differed markedly with substrate angle, as shown by NMDS ordination and CAP analysis (Fig. 7). More than half of invertebrate species recorded were present only on rock walls, and none were present only on horizontal substrates. Horizontal substrates were significantly more variable in species composition (Fig. 7A), which could be ascribed to the lower abundance of invertebrates on the horizontal; however, this variability may also indicate that horizontally-oriented invertebrate communities may be more variable in space and time than those on vertical walls. Solitary ascidians in general were not more likely to be found on horizontal substrate compared to colonial forms, although 2 solitary species, Molgula citrina DeKay and Dendrodoa carnea Agassiz, were often found on horizontal substrate. Anemones (Cnidaria) were particularly common on horizontal substrates; possibly their ability to move, albeit slowly, enables them to avoid competitive exclusion by fast-growing algae or burial by shifting sediment. The sponge Isodictya palmata was also common on horizontal substrates; its upright morphology may allow this species to shed sediment. However, it is difficult to generalize about which invertebrate species are confined largely to walls. Encrusting species are sometimes thought to be more vulnerable to sedimentation than upright forms (e.g. Jackson 1977), but some encrusting colonial tunicates (including the native species Didemnum albidum) were common on horizontal substrate. Abundance of tube complex, mussels, the encrusting algae Peysonnalia sp. and corallines did not differ with substrate angle. Tube complex is a transient assemblage composed of tube-building amphipods and polychaete worms, and its abundance may vary with higher temporal frequency than does disturbance or algal overgrowth on horizontal platforms (Sebens 1986a). Mussels form extensive beds on horizontal substrates, and depend on grazers to remove overgrowing algae that would otherwise cause dislodgement of mussels in storms (Witman 1987). Encrusting algae, particularly corallines, can be very resistant to burial by sediment or other organisms (Sebens 1986a, Airoldi 2000), may benefit from disturbance (Steneck 1982), and are well adapted to the lowlight conditions on walls by virtue of their encrusting sheet morphology (Steneck 1986). However, coralline algae, like sessile invertebrates, are vulnerable to overgrowth by macroalgae, and often rely on grazing or scouring to prevent overgrowth (Steneck 1986). Residing on walls may also prevent macroalgal overgrowth of corallines, as is the case for invertebrates (Miller \& Etter 2008). Experimental work on these taxa may provide valuable insights into their relative success on vertical walls compared to other algae. 
The only consumers that showed consistent patterns with substrate orientation were the chitons and the asteroid Henricia sanguinolenta, both of which were more common on vertical walls (Fig. 6). Chitons graze on coralline and other crustose algae, which were common on vertical surfaces (Fig. 5). The limpet Tectura testudinalis, which also grazes corallines, was not more abundant on vertical walls. This difference may reflect lower abundance of the coralline Clathromorphum circumscriptum Foslie, T. testudinalis's preferred food (Steneck 1982) on vertical surfaces (Sebens 1986a), probably due to the high light requirements of this species (Adey 1965). Coralline communities on GOM walls are typically dominated by the genera Phymatolithon and Lithothamnion (Sebens 1986a).

The asteroid Henricia sanguinolenta, a suspension feeder, also feeds on sessile invertebrates, especially sponges, ascidians, and bryozoans (Sheild 1990, Sheild \& Witman 1993). Numerous $H$. sanguinolenta were observed feeding on invertebrates, particularly the ascidians Aplidium glabrum Verrill and Didemnum albidum, and opening patches of bare space at the centimeter to decimeter scale, suggesting that $H$. sanguinolenta may be an important space-freeing predator in these communities (R. Miller unpubl. data). $H$. sanguinolenta was more abundant on vertical walls where its prey species were most abundant. Positioning on a wall may also allow this asteroid to suspension feed more effectively, similar to sessile suspension feeders (Leichter \& Witman 1997). Suspension feeding is thought to be the main nutritional mode of the genus Henricia, with some species at least supplementing their diet by feeding on invertebrates. Indeed, Henricia often suspension feeds from the tips of erect sponges and bryozoans (reviewed in Sheild 1990).

The similarity in shared species across our sites, which span nearly the entirety of the GOM, indicates the Gulf can be considered an intact biogeographic region, at least with respect to shallow-water sessile invertebrates. Indeed, sites were more similar to each other, on average, than were quadrats within a given site. This is consistent with the relatively homogenous sea surface temperatures and tidal amplitudes within the Gulf (Schmidt et al. 2008). The greater heterogeneity within sites compared to between sites suggest that local interactions might be more important than between-site processes in structuring shallow sessile communities within this region.

Do the data presented here shed light on what mechanisms are most important in shaping the pattern of high invertebrate abundance and diversity on rock walls in the GOM? The factors commonly invoked as important are shading, grazing, sedimentation, physical disturbance, and water flow (reviewed in Witman \& Dayton 2001). Shading likely affects invertebrates via competition for space with algae (Miller \& Etter 2008). Unfortunately, we cannot simply compare algal and invertebrate cover using the same quadrats, since the 2 measurements would necessarily be correlated (for discussion, see Underwood 1997). It is clear, however, that macroalgae are typically much more abundant on horizontal surfaces (Fig. 5). The only 2 sites where this pattern is absent are Cutler and Thrumcap. We have discussed the abundance of the colonial invasive ascidian Didemnum vexillum, which commonly overgrows macroalgae (Bullard et al. 2007, R. Miller pers. obs.) on horizontal substrate at Thrumcap. Cutler had the highest urchin densities of any site, and both macroalgae and invertebrates were rare on horizontal surfaces (Fig. 5). These low abundances of invertebrates and algae were likely due to urchin grazing. Urchin density was negatively correlated with invertebrate cover on horizontal rocky bottoms, suggesting that urchin grazing may be an important factor limiting invertebrates to vertical walls; however this phenomenon may be limited to areas with exceptionally high urchin densities. We have no data on sedimentation, physical disturbance, and water flow to compare between sites, which are all exposed and likely similar hydrodynamically. Nevertheless, vertical walls at 3 sites, Thrumcap, Bunker Point, and Wood Island, had considerably lower richness than the other sites (Fig. 2). We have discussed the dominance of colonial invasive ascidians driving this pattern at Thrumcap. The other 2 were the only sites outside river mouths: Bunker Point receives outflow from the St. Croix River, while Wood Island is near the mouth of the Saco River. Thus, higher levels of sedimentation and disturbance in the form of variable salinity (Witman \& Grange 1998) likely prevail at these sites. Overall, the most consistent pattern among the sites was higher abundance and richness of sessile invertebrates on vertical walls, and the much higher abundance of algae on horizontal platforms.

Communities on rock walls in the GOM are drastically different from those on adjacent horizontal platforms at 10 to 12 meter depth. Walls were dominated (percent cover) by invertebrates and supported 4 times as many species as nearby horizontal platforms. The segregation of algae and invertebrates between horizontal platforms and vertical walls, respectively, was the most consistent difference between these 2 habitats. This strong pattern, combined with experimental work showing that shading of horizontal rock was sufficient to cause a switch to invertebrate-dominated communities (Miller \& Etter 2008), suggests that competition with faster-growing algae and/or the impact of the algal-associated fauna (e.g. micropredators) are the primary mechanisms limiting invertebrates to vertical walls in the GOM. The omnivorous urchin Strongylocentrotus droebachiensis may also play a role 
where it is abundant by regulating invertebrate cover on horizontal rock. Whatever the mechanisms active at an individual site, substrate angle clearly plays an important role in maintaining diversity of both plants and animals in subtidal communities.

On land, rocky cliffs can be important refuges from anthropogenic disturbance, and may harbor remarkable floral and faunal diversities (reviewed in Larson et al. 2000). Subtidal rock walls in the GOM are hotspots of sessile invertebrate diversity and abundance. If the enhanced diversity of vertical walls is repeated in other regions of the world's oceans, as suggested by anecdotal evidence (references above), coastal areas with greater topographic complexity are likely to support much more diverse invertebrate assemblages. More importantly, the structure, composition and function of subtidal ecosystems will be influenced by the degree of topographic heterogeneity.

Acknowledgements: Northeastern University's Marine Science Center and the Darling Marine Center graciously provided facilities and support. P. Miller enthusiastically helped in the field. J. Witman, K. Sebens, E. Gallagher, and M. Rex contributed valuable discussion and comments. Comments by 3 anonymous reviewers and $M$. Pedersen improved the manuscript. This study was supported by NSF Bio-Ocean grant 0017839 to R.J.E. and R.J.M., and by grants to R.J.M. from PADI Project AWARE, UMass Boston, and Sigma Xi.

\section{LITERATURE CITED}

Adey WH (1965) The genus Clathromorphum (Corallinaceae) in the Gulf of Maine. Hydrobiologia 26:539-574

Airoldi L (2000) Effects of disturbance, life history, and overgrowth on coexistence of algal crusts and turfs. Ecology 81:798-814

Anderson MJ (2004) PERMDISP: a FORTRAN computer program for permutational analysis of multivariate dispersions using permutation tests. Dept of Statistics, University of Auckland

Anderson MJ, Robinson J (2003) Generalised discriminantanalysis based on distances. Austral NZ J Stat 45:301-318

> Anderson MJ, Willis TJ (2003) Canonical analysis of principal coordinates: a useful method of constrained ordination for ecology. Ecology 84:511-525

Baynes TW (1999) Factors structuring a subtidal encrusting community in the southern Gulf of California. Bull Mar Sci 64:419-450

Beck DD, Jennings RD (2003) Habitat use by gila monsters: the importance of shelters. Herp Monog 17:111-129

Berman J, Harris L, Lambert W, Buttrick M, Dufresne M (1992) Recent invasions of the Gulf of Maine: three contrasting ecological histories. Conserv Biol 6:435-441

Bertness MD (1999) The ecology of Atlantic shorelines. Sinauer Associates, Sunderland, MA

Bruno JF, Witman JD (1996) Defense mechanisms of scleractinian cup corals against overgrowth by colonial invertebrates. J Exp Mar Biol Ecol 207:229-241

Bullard SG, Lambert G, Carman MR, Byrnes J and others (2007) The colonial ascidian Didemnum sp. A: current distribution, basic biology, and potential threat to marine communities of the northeast and west coasts of North America. J Exp Mar Biol Ecol 342:99-108

Casgrain P, Legendre P (2001) The R package for multivariate and spatial analysis, version $4.0 \mathrm{~d} 5$ - user's manual. University of Montreal

Chen YC, Hwang WH, Chao A, Kuo CY (1995) Estimating the number of common species. Analysis of the number of common bird species in Ke-Yar Stream and Chung-Kang Stream. J Chin Stat Assoc 33:373-393

Clark DA, Clark DB, Sandoval RM, Castro MV (1995) Edaphic and human effects on landscape-scale distributions of tropical rain forest palms. Ecology 76:2581-2594

Colwell RK (1997) User's guide to EstimateS 5: statistical estimation of species richness and shared species from samples, Version 5.0.1 University of Connecticut, Storrs

Colwell RK, Coddington JA (1994) Estimating terrestrial biodiversity through extrapolation. Philos Trans R Soc Lond B 345:101-118

Coyer J, Steller D, Witman JD (1999) The underwater catalog: a guide to methods in underwater research. Shoals Marine Laboratory, Ithaca, NY

> Davis MA, Pelsor M (2001) Experimental support for a resource-based mechanistic model of invasiblity. Ecol Lett $4: 421-428$

Dayton PK (1975) Experimental studies of algal canopy interactions in a sea otter-dominated kelp community at Amchitka Island, Alaska. Fish Bull 73:230-237

Denny MW (1988) Biology and the mechanics of the waveswept environment. Princeton University Press, Princeton, NJ

Genovese SJ, Witman JD (1999) Interactive effects of flow speed and particle concentration on growth rates of an active suspension feeder. Limnol Oceanogr 44: 1120-1131

Gili JM, Coma R (1998) Benthic suspension feeders: their paramount role in littoral marine food webs. Trends Ecol Evol 13:316-321

Golikov AN, Scarlato OA (1968) Vertical and horizontal distribution of biocoenoses in the upper zones of Japan and Okhotsk Seas and their dependence on the hydrological system. Sarsia 34:109-116

Gotelli NJ (1987) Spatial and temporal patterns of reproduction, larval settlement, and recrutment of the compound ascidian Aplidium stellatum. Mar Biol 94:45-51

> Graham K, Sebens KP (1996) The distribution of marine invertebrate larvae near vertical surfaces in the rocky subtidal zone. Ecology 77:105-121

> Irving AD, Connell SD (2002) Sedimentation and light penetration interact to maintain heterogeneity of subtidal habitats: algal versus invertebrate dominated assemblages. Mar Ecol Prog Ser 245:83-91

> Jackson JBC (1977) Competition on marine hard substrata: the adaptive significance of solitary and colonial strategies. Am Nat 111:743-767

Knott NA, Underwood AJ, Chapman MG, Glasby TM (2004) Epibiota on vertical and on horizontal surfaces on natural reefs and on artificial structures. J Mar Biol Assoc UK 84: $1117-1130$

> Lambert G (2009) Adventures of a sea squirt sleuth: unraveling the identity of Didemnum vexillum, a global ascidian invader. Aquatic Invasions 4:5-28

Larson DW, Matthes U, Kelly PE (2000) Cliff ecology: pattern and process in cliff ecosystems. Cambridge University Press, Cambridge

> Leichter JJ, Witman JD (1997) Water flow over subtidal rock walls: relation to distributions and growth rates of sessile suspension feeders in the Gulf of Maine - water flow and growth rates. J Exp Mar Biol Ecol 209:293-307 
Little C, Kitching JA (1996) The biology of rocky shores. Oxford University Press, Oxford

Lundalv T (1971) Quantitative studies on rocky-bottom biocoenoses by underwater photogrammetry. Thalassia Jugoslavica 7:201-208

Magurran AE (2004) Measuring biological diversity. Blackwell Publishing, Oxford

Mathieson AC, Penniman CA, Harris LG (1991) Northwest Atlantic rocky shore ecology. In: Mathieson AC, Nienhuis $\mathrm{PH}$ (eds) Intertidal and littoral ecosystems. Elsevier, Amsterdam

Miller RJ, Etter RJ (2008) Shading facilitates invertebrate dominance of subtidal vertical walls. Ecology 89:452-462

Noble J, Logan A, Webb G (1976) The recent Terebrutulina community in the rocky subtidal zone of the Bay of Fundy, Canada. Lethaiu 9:1-17

Ohmann JL, Spies TA (1998) Regional gradient analysis and spatial pattern of woody plant communities of Oregon forests. Ecol Monogr 68:151-182

Osman RW, Whitlatch RB (2007) Variation in the ability of Didemnum sp. to invade established communities. J Exp Mar Biol Ecol 342:40-53

Parvinen K, Meszéna G (2009) Disturbance-generated nichesegregation in a structured metapopulation model. Evol Ecol Res 11:651-666

> Pequegnat WE (1964) The epifauna of a California siltstone reef. Ecology 45:272-282

Porter WP, Sabo JL, Tracy CR, Reichman OJ, Ramankutty N (2002) Physiology on a landscape scale: plant-animal interactions. Integr Comp Biol 42:431-453

Ramsey F, Schafer D (2002) The statistical sleuth: a course in methods of data analysis. Duxbury Press, Pacific Grove, CA

Ricketts EF, Calvin J, Hedgpeth JW revised by Phillips DW (1985) Between Pacific tides. Stanford University Press, Stanford, CA

Schmidt PS, Serrao EA, Pearson GA, Riginos C and others (2008) Ecological genetics in the north Atlantic: environmental gradients and adaptation at specific loci. Ecology 89:S91-S107

Sebens KP (1986a) Spatial relationships among encrusting marine organisms in the New England subtidal zone. Ecol Monogr 56:73-96

Sebens KP (1986b) Community ecology of vertical rock walls in the Gulf of Maine, USA: small-scale processes and alternative community states. In: Moore PG, Seed R (eds) The Ecology of Rocky Coasts. Hodder and Stoughton Educational Press, London, p 346-371

Sheild CJ (1990) Predation by the sea star, Henricia sanguinolenta (Echinodermata, Asteroidea), with special attention to its effect on sponges. MS thesis, Northeastern University, Boston, MA

Sheild CJ, Witman JD (1993) The impact of Henricia sanguinolenta (O. F. Müller) (Echinodermata Asteroidea) predation on the finger sponges, Isodictya spp. J Exp Mar Biol Ecol 166:107-133

Simoncini M, Miller RJ (2007) Feeding preference of Strongylocentrotus droebachiensis (Echinoidea) for a dominant native ascidian Aplidium glabrum, relative to the invasive

Editorial responsibility: Morten Pedersen,

Roskilde, Denmark ascidian Botrylloides violaceus. J Exp Mar Biol Ecol 342: 93-98

Smith F, Witman JD (1999) Species diversity in subtidal landscapes: maintenance by physical processes and larval recruitment. Ecology 80:51-69

Stefaniak L, Lambert G, Gittenberger A, Zhang H, Lin S, Whitlatch RB (2009) Genetic conspecificity of the worldwide populations of Didemnum vexillum Kott 2002. Aquatic Invasions 4:29-44

Steneck RS (1982) A limpet-coralline alga association: adaptations and defenses between a selective herbivore and its prey. Ecology 63:507-522

Steneck RS (1986) The ecology of coralline algal crusts: convergent patterns and adaptive strategies. Annu Rev Ecol Syst 17:273-303

Thompson JD (1991) The biology of an invasive plant. Bioscience 41:393-401

Underwood AJ (1997) Experiments in ecology: their logical design and interpretation using analysis of variance. Cambridge University Press, Cambridge

Underwood AJ, Chapman MG, Connell SD (2000) Observations in ecology: you can't make progress on processes without understanding the patterns. J Exp Mar Biol Ecol 250:97-115

Uriz MJ, Maldonado M, Turon X, Marti R (1998) How do reproductive output, larval behavior, and recruitment contribute to adult spatial patterns in Mediterranean encrusting sponges? Mar Ecol Prog Ser 167:137-148

Vance RR (1988) Ecological succession and the climax community on a marine subtidal rock wall. Mar Ecol Prog Ser 48:125-136

Weinberg S (1978) Mediterranean octocorallian communities and the abiotic environment. Mar Biol 49:41-57

Weiss SB, Murphy DD, White RR (1988) Sun, slope and butterflies: topographic determinants of habitat quality for Euphydras editha. Ecology 69:1486-1496

Whittaker RH (1960) Vegetation of the Siskiyou Mountains, Oregon and California. Ecol Monogr 30:279-338

- Witman JD (1987) Subtidal coexistence: storms, grazing, mutualism and the zonation of kelps and mussels. Ecol Monogr 57:167-187

Witman JD, Cooper RA (1983) Disturbance and contrasting patterns of population structure in the brachiopod Terebra tulina septentrionalis (Couthouy) from two subtidal habitats. J Exp Mar Biol Ecol 73:57-79

Witman JD, Dayton PK (2001) Rocky subtidal communities. In: Bertness M, Gaines S, Hay M, (eds) Marine community ecology. Sinauer, Sunderland, MA

Witman JD, Grange KR (1998) Links between rain, salinity, and predation in a rocky subtidal community. Ecology 79 : 2429-2447

Witman JD, Sebens KP (1990) Distribution and ecology of sponges at a subtidal rock ledge in the central Gulf of Maine. In: Rutzler K (ed) New Perspectives in Sponge Biology. Smithsonian Institution Press, Washington, DC

> Witman JD, Etter RJ, Smith F (2004) The relationship between regional and local species diversity in marine benthic communities: a global perspective. Proc Natl Acad Sci USA 101:15664-15669

Submitted: August 8, 2010; Accepted: Januar 10, 2011

Proofs received from author(s): March 3, 2011 